\title{
Referees 2016
}

Published online: 31 January 2017

(C) Springer-Verlag Berlin Heidelberg 2017

We thank those who have given their time to referee for Diabetologia, all of whom are listed on our website (http://www. diabetologia-journal.org/referees.html). Special thanks are due to our key referees, listed below, who have each provided at least three timely and well-rated reports over the past year.

\begin{tabular}{|c|c|c|c|}
\hline Abderrahmani A; Lille & Del Prato S; Pisa & Hanssen N; Maastricht & Lovshin J; Ontario \\
\hline Advani A; Toronto & Delibegovic M; Aberdeen & Harris R; London & Lowe W; Chicago \\
\hline Allred ND; Winston Salem & Desoye G; Graz & Hattersley A; Exeter & Lystig T; Ridgefield \\
\hline \multirow[t]{2}{*}{ Arner P; Stockholm } & Donath MY; Zürich & Heinzmann S; Neuherberg & \\
\hline & Dotta F; Siena & Hesselink M; Maastricht & Ma RCW; Hong Kong \\
\hline Belfiore A; Catanzaro & & Home P; Newcastle upon & MacDonald P; Edmonton \\
\hline Belgardt B; Düsseldorf & Ekelund U; Cambridge & Tyne & Malecki M; Krakow \\
\hline Bell J; Melbourne & Elding Larsson H; Malmö & Horváth VJ; Budapest & Malik RA; Manchester \\
\hline Biessels G; Utrecht & Eliasson B; Gothenburg & & Malin S; Charlottesville \\
\hline Bolli GB; Perugia & Eringa EC; Amsterdam & Imai Y; Iowa City & Marchetti P; Pisa \\
\hline Bonadonna RC; Parma & Evans M; Cambridge & & Maresh M; Manchester \\
\hline Bonner-Weir S; Boston & & Jandeleit-Dahm K; Melbourne & Mari A; Padua \\
\hline Bouchard L; Montreal & Fadini GP; Padua & Jansson L; Uppsala & Matveyenko AV; Rochester \\
\hline Brozinick JT; Indianapolis & Fall T; Uppsala & Jörns A; Hanover & McElduff A; Sydney \\
\hline Buchanan TA; Los Angeles & Feig D; Toronto & Johnson JD; Vancouver & Meyre D; Lille \\
\hline Butler PC; Los Angeles & $\begin{array}{l}\text { Foley JE; East Hanover } \\
\text { Folli F; San Antonio }\end{array}$ & Jones AG; Exeter & $\begin{array}{l}\text { Mingrone G; Rome } \\
\text { Monnier V; Cleveland }\end{array}$ \\
\hline Caicedo A; Miami & Freathy R; Exeter & Karalliedde J; London & Morgan NG; Exeter \\
\hline Campbell-Thompson M; & Freeman D; Glasgow & Kay TW; Fitzroy & Mulder H; Malmö \\
\hline Gainesville & Froguel P; Lille & Kerr-Conte J; Lille & Murphy HR; Cambridge \\
\hline Canto C; Lausanne & & Keymeulen B; Brussels & Muscelli E; São Paulo \\
\hline Carlsson P; Uppsala & Gallwitz B; Tübingen & Khoury J; Cincinnati & \\
\hline Carty D; Glasgow & Garcia-Ocana A; Pittsburgh & Kilpeläinen TO; Cambridge & Nadler JL; Norfolk \\
\hline Catalano P; Cleveland & Gault VA; Coleraine & King G; Boston & Nascimento E; Maastricht \\
\hline Chan O; New Haven & Gesualdo L; Bari & Kitamura T; Maebashi & Nauck MA; Bochum \\
\hline Chu A; Boston & Gilon P; Brussels & Knip M; Helsinki & Nielsen J; Copenhagen \\
\hline Colhoun H; Edinburgh & Grace M; Melbourne & Kushner JA; Houston & Nolan CJ; Woden \\
\hline \multirow[t]{2}{*}{ Cooper ME; Melbourne } & Greenbaum CJ; Seattle & & Nuutila P; Turku \\
\hline & & Landgraf R; Munich & \\
\hline Dauriz M; Boston & Häring H-U; Tübingen & Lau B; Baltimore & Oliver N; London \\
\hline Davidson H; Denver & Hajduch E; Paris & Leibiger I; Stockholm & Owen KR; Oxford \\
\hline
\end{tabular}


Panasyuk G; Paris

Perseghin G; Milan

Pflüger P; Garching

Powers AC; Nashville

Preiss D; Oxford

Prieur X; Nantes

Pugliese A; Miami

Redondo M; Houston

Ren J; Laramie

Renehan A; Manchester

Richter E; Copenhagen

Ringholm L; Copenhagen

Roep BO; Duarte

Rorsman P; Oxford
Ross G; Sydney

Rossing P; Gentofte

Rutter GA; London

Schmitz G; Regensburg

Sharp S; Cambridge

Shearer J; Calgary

Simmons D; Cambridge

Simmons RC; Philadelphia

Söderberg S; Umeå

Solini A; Pisa

Speier S; Dresden

Staels B; Lille

Steck AK; Aurora

Stettler C; Bern
Stienstra R; Nijmegen

Tanti J; Nice

Taylor R; Newcastle upon

Tyne

Thivolet C; Lyons

Umpierrez G; Atlanta

van de Bunt $\mathrm{M}$; Oxford

Vas P; London

Verchere B; Vancouver

Vettor R; Padua

Vidal-Puig AJ; Cambridge

von Herrath MG; La Jolla
Wang-Sattler R; Neuherberg

Weedon M; Exeter

Weigert C; Tübingen

Weissgerber T; Rochester

Welsh N; Uppsala

Wicksteed B; Chicago

Williams AJK; Bristol

Woo M; Toronto

Zaccardi F; Leicester

Zerbini G; Milan

Zheng Y; Boston 